\title{
A Review of Convergence Analysis of Particle Swarm Optimization
}

\author{
Dong ping $\operatorname{Tian}^{1,2}$ \\ ${ }^{1}$ Institute of Computer Software, Baoji University of Arts and Sciences, \\ Baoji, Shaanxi, 721007, China \\ ${ }^{2}$ Institute of Computational Information Science, Baoji University of Arts and \\ Sciences, Baoji, Shaanxi, 721007, China \\ tdp211@163.com
}

\begin{abstract}
Particle swarm optimization (PSO) is a population-based stochastic optimization originating from artificial life and evolutionary computation. PSO is motivated by the social behavior of organisms, such as bird flocking, fish schooling and human social relations. Its properties of low constraint on the continuity of objective function and ability of adapting to the dynamic environment make PSO become one of the most important swarm intelligence algorithms. However, compared to the various version of modified PSO and the corresponding applications in many domains, there has been very little research on the PSO's convergence analysis. So the current paper, to begin with, elaborates the basic principles of standard PSO. Then the existing work on the convergence analyses of PSO in the literatures is thoroughly surveyed, which plays an important role in establishing the solid theoretical foundation for PSO algorithm. In the end, some important conclusions and possible research directions of PSO that need to be studied in the future are proposed.
\end{abstract}

Keywords: PSO, Swarm intelligence, Constriction coefficient, Limit, Differential equation, Difference equation, $Z$ transformation, Bilinear transformation, Routh criterion

\section{Introduction}

Particle swarm optimization is a swarm-based evolutionary algorithm. PSO is first introduced by Eberhart and Kennedy and used for optimization of continuous non-linear functions [1-3]. It is similar to other population-based evolutionary algorithms in that the algorithm is initialized with a population of random solutions, such as ant colony optimization (ACO) and genetic algorithms (GA). It is unlike most of other population-based evolutionary algorithms, however, in that PSO is motivated by the simulation of social behavior instead of survival of the fittest, and each candidate solution is associated with a velocity [4]. Due to the convenience of realization and promising optimization ability, PSO has been paid much attention to by researchers since its advent. So far, PSO has been successfully applied in solving various optimization problems, such as function optimization, training artificial neural networks [5], pattern classification [6] and fuzzy system control, etc. particularly for the problems under the multidimensional and dynamic conditions. Therefore, to some extent, PSO is not only suitable for science researches, but also for engineering applications particularly. Over the years, researches of PSO have been focused on two aspects. The first one is the performance improvement of PSO by modifying its parameters, increasing population diversity and fusing with other optimization methods. The second is the applicati- ons of PSO in different areas. In comparison with the hot discussion on the two 
aspects mentioned above, little attention has been paid to the theoretical analysis and proof. However, it is very important to understand the PSO's theoretical foundations, which can further help to master how PSO works. So in this paper, the convergence analysis of PSO is summarized systematically. Meanwhile, some important conclusions and research directions of PSO that need to be studied in the future are proposed.

The remainder of the text is organized as follows. Section 2 introduces the standard PSO. In Section 3, the existing convergence analyses of PSO algorithm are summarized in detail by six subsections, including convergence analysis with constriction coefficient, limit, differenttial equation, matrix, difference equation and $Z$ transformation. Finally, the paper ends in obtaining some important conclusions and pointing out some possible future research directions to be studied in Section 4.

\section{Standard PSO}

PSO is originally designed by Kennedy and Eberhart [1]. It is inspired by natural concepts such as bird flocking and fish schooling. In PSO system, each candidate solution is called a particle, each particle moves in the search space with a velocity that is dynamically adjusted according to the corresponding particle's experience and the particle's companions' experience. Mathematically, the particles are manipulated according to the following equations [2]:

$$
\begin{aligned}
& v_{i d}(t+1)=\omega \times v_{i d}(t)+c_{1} \times r_{1} \times\left[p_{i d}(t)-x_{i d}(t)\right]+c_{2} \times r_{2} \times\left[p_{g d}(t)-x_{i d}(t)\right] \\
& x_{i d}(t+1)=x_{i d}(t) t e x \times v_{i d}(t+
\end{aligned}
$$

where $c_{1}$ and $c_{2}$ are positive constants, called acceleration coefficients. $r_{1}$ and $r_{2}$ are two random functions in the range [0,1]. $\omega$ is the inertia weight, it has characteristics that are reminiscent of the temperature parameter in the simulated annealing (SA). A large inertia weight facilitates a global exploration while a small inertia weight facilitates a local exploitation. The $i$-th particle is represented as $X_{i}=\left(x_{i 1}, x_{i 2}, \ldots, x_{i D}\right)$. The best previous position (the position giving the best fitness value) of the $i$-th particle is recorded and represented as $P_{i}=\left(p_{i l}, p_{i 2}, \ldots, p_{i D}\right)$. The index of the best particle among all the particles in the population is represented by the symbol $g$. The rate of the position change (velocity) for particle $i$ is represented as $V_{i}=\left(v_{i l}, v_{i 2}, \ldots, v_{i D}\right)$. During the update, the maximum velocity of each dimension of a particle is restricted to $v_{\max }$, whose coordination of every dimension is also restricted to the permission scope. $D$ represents the dimension of the search space. Figure 1 illustrates the moving principle of particles in the swarm.

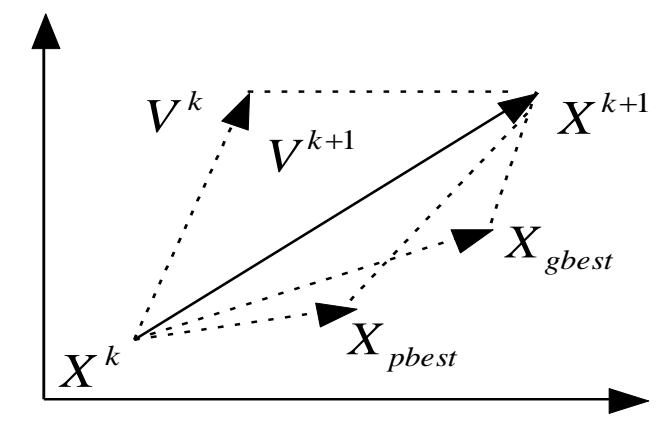

Figure 1. Moving principle of particles 
In equation (1), the first part is the previous velocity of the particle. The second is the "cognitive" part, representing the exploiting of its own experience, where $c_{l}$ is individual factor. And the third denotes the "social" part, representing the shared information and mutual cooperation among the particles, where $c_{2}$ is societal factor. Alternatively, the procedure of the standard PSO algorithm is illustrated as follows.

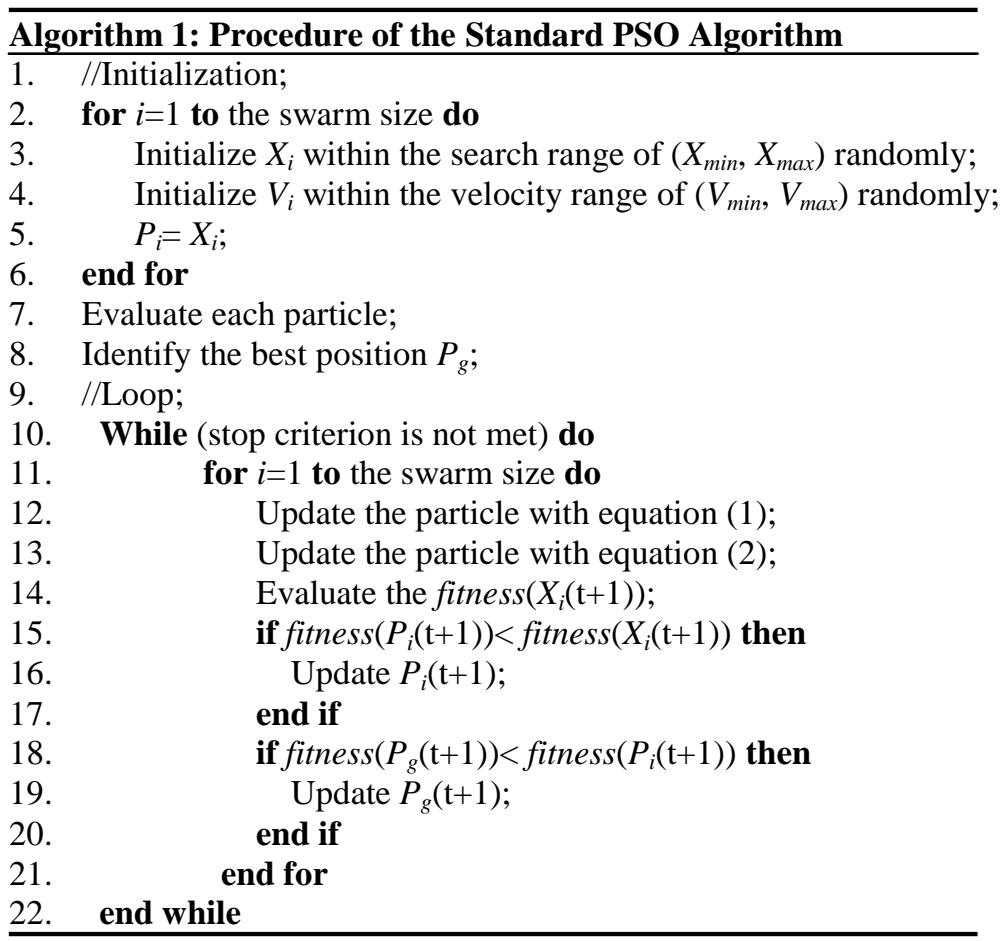

\section{Convergence Analyses of PSO}

Since PSO is put forward in 1990s, it has become the focus in optimization community and has been widely applied in many fields. Much attention has been paid to the improvements of PSO itself, such as the improvement of its parameters, including the inertia weight and convergence factor, the improvement of the update formula based on the velocity-position, the improvement based on the topology of particle swarms, the improvement based on the evolutionary mechanism of the genetic algorithm, including selection, crossover and mutation, and the improvement based on the integration of other approaches, viz., the so-called hybrid soft computing (HSC). However, very little research on the PSO's convergence has been studied so far, which plays a crucial role in establishing the solid theoretical foundation for PSO algorithm. So this paper, from another perspective, thoroughly reviews and analyzes the convergence of PSO in the existing literature, the goal is to provide references and suggestions for PSO researchers to establish a solid theoretical basis. The details of them will be described in the following subsections respectively.

\subsection{Convergence Analysis with Constriction Coefficient}

The parameters appearing in equations (1) and (2) are primarily analyzed by Clerc in [7]. Although there are obvious defects, it is very important for researchers to study the convergence of PSO. The highly simplified deduction process is as follows. First, redefining $P_{i d} \leftarrow\left(c_{l} p_{i d}+c_{2} p_{g d}\right) /\left(c_{1}+c_{2}\right)$, then equation (1) can be simplified as $v_{i d} \quad(t+1)=v_{i d}$ 
$(t)+c\left(p_{i d}(t)-x_{i d}(t)\right)$, where $c=c_{1}+c_{2}$. At this time, the PSO system can be further simplified by considering a one-dimensional problem space and again by reducing the population to one particle. Meanwhile, assume that both $P_{g d}$ and $c$ are constants. Then equations (1) and (2) can be represented as follows ( $p$ and $c$ are constants):

$$
\left\{\begin{array}{c}
v(t+1)=v(t)+c(p-x(t)) \\
x(t+1)=x(t)+v(t+1)
\end{array}\right.
$$

The constriction condition of particles' stability can be obtained via the stable analysis of equation (3), and the important conclusion is drawn as below. When the parameters appearing in equations (1) and (2) meet the constriction condition,

$$
v_{i d}(t+1)=K \times\left[v_{i d}(t)+c_{1} \times r_{1} \times\left(p_{i d}(t)-x_{i d}(t)\right)+c_{2} \times r_{2} \times\left(p_{g d}(t)-x_{i d}(t)\right)\right]
$$

where $K=2 /\left|2-c-\sqrt{c^{2}-4 c}\right|$. If $c=c_{1}+c_{2}>4$, then the particle's trajectory in the PSO system is stable under these assumption conditions.

\subsection{Convergence Analysis with Limit}

The relation between the convergence and the variance of the population's fitness is manifested in [8]. First, defining the variance of the population's fitness as below:

$$
\sigma^{2}=\frac{1}{N} \sum_{i=1}^{N}\left(\frac{f_{i}-f_{a v g}}{f}\right)^{2}
$$

where $N$ represents the number of particles, $f_{i}$ is the fitness of the $i$-th particle, $f_{\text {avg }}$ is the current average fitness of the swarm, $f$ is the normalized calibration factor to confine $\sigma^{2}$. The value of $f$ is derived from:

$$
f=\max \left\{1, \max \left\{\left|f_{i}-f_{\text {avg }}\right|\right\}\right\}, i \in[1, N]
$$

The definition of $\sigma^{2}$ shows that it presents the convergence degree of all the particles in the swarm. A smaller $\sigma^{2}$ presents a better convergence. On the contrary, the particle swarm is still in the random searching. Then the particle's convergence in the swarm can be defined as:

$$
\lim _{t \rightarrow+\infty} X(t)=P
$$

where $X(t)$ denotes the position of a particle at time $t$, and $p$ is the arbitrary position of the whole searching space. This definition demonstrates that the particles will finally settle in some fixed position $p$ in the searching space. Alternatively, the following conclusion can be derived through strict mathematical derivation by [9].

$$
\lim _{t \rightarrow+\infty} X(t)=\alpha \times p \text { Best }+(1-\alpha) \times g \text { Best }
$$

where $\alpha=c_{1} /\left(c_{1}+c_{2}\right), c_{1}$ and $c_{2}$ are acceleration coefficients in equation (1), pBest denotes the individual extremum of this particle while gBest is the global extremum of the particle swarm.

Based on the conclusion given above, Kennedy and Eberhart [1] further demonstrate that if PSO plunges into premature convergence or global convergence, the particles in the swarm will gather in one or some special position, and the variance of the population's fitness is zero 


\subsection{Convergence Analysis with Differential Equation}

The relation between the inertia weight and the particle's velocity is described in [10]. Like other methods of convergence analysis, first, supposing that the problem space is onedimensional and there is only one particle in the swarm. Then $p_{i}=p_{g}$ can be obtained. At the same time, assuming that $\Phi_{l}=r_{1} c_{1}, \Phi_{2}=r_{2} c_{2}$ and $\Phi=\Phi_{1}+\Phi_{2} . y_{i}$ can be denoted by $p_{i}-x_{i}$. Thus equations (1) and (2) can be simplified as:

$$
\begin{aligned}
& v(t+1)=\omega v(t)+\varphi y(t) \\
& y(t+1)=-\omega v(t)+(1-\varphi) y(t)
\end{aligned}
$$

The following formula can be derived by iterating equations (9) and (10) and eliminating variable $y$ :

$$
v(t+2)+(\varphi-1-\omega) v(t+1)+\omega v(t)=0
$$

Making equation (11) be continuous and a second-order differential equation is derived:

$$
\frac{\partial^{2} v}{\partial t^{2}}+\operatorname{In}\left(e_{1} e_{2}\right) \frac{\partial v}{\partial t}+\operatorname{In}\left(e_{1}\right) \operatorname{In}\left(e_{2}\right) v=0
$$

where $e_{1}$ and $e_{2}$ are the roots of the quadratic equation $\lambda^{2}+(\varphi-1-\omega) \lambda+\omega=0$. More precisely, $e_{1}=(\omega+1-\varphi+\sqrt{\Delta}) / 2, e_{2}=(\omega+1-\varphi-\sqrt{\Delta}) / 2$ where $\Delta=(\omega+1-\varphi)^{2}-4 \omega$.Thus, the general solution of the differential equation (12) is as follows:

$$
v(\mathrm{t})=k_{1} e_{1}^{t}+k_{2} e_{2}^{t}
$$

By further reasoning,

$$
y(t)=\left[k_{1} e_{1}^{t}\left(e_{1}-\omega\right)+k_{2} e_{2}^{t}\left(e_{2}-\omega\right)\right] / \varphi
$$

where $k_{1}=\left(-\varphi y(0)-\left(\omega-e_{2}\right) v(0)\right) /\left(e_{2}-e_{1}\right), k_{2}=\left(\varphi y(0)+\left(\omega-e_{1}\right) v(0)\right) /\left(e_{2}-e_{1}\right)$. From equations $(13)$ and (14), if $t$ tends to infinity and $\max \left(\left|e_{1}\right|,\left|e_{2}\right|\right)<1$, then $v(t)$ and $y(t)$ will tend to infinity. That is to say, the PSO converges at the time. If $\omega>0.5 \varphi-1$, then $\max \left(\left|e_{1}\right|,\left|e_{2}\right|\right)<1$; While if $\omega>0.5 \times$ $\left(c_{1}+c_{2}\right)-1$, then $\omega>0.5 \varphi-1$ holds and the PSO do converge.

By comparison, the convergence analysis method referred in [10] is actually very similar to that in [7]. Their slight differences mainly lie in two aspects. The first one is that $\omega$ in [7] is set to 1 whereas in [10] employed by itself. The second is that the convergence condition in [10] is directly given while in [7] the basic explicit representation, a posteriori proof, general implicit and explicit representations, and the transformation from ER to IR are described in detail respectively.

\subsection{Convergence Analysis with Matrix}

On the basis of [11] for the convergence analysis of particle's trajectory, the influence of particle's velocity on the convergence is further discussed in [12]. First, assume that $\phi_{i, j}^{(k)}(t)=c_{k} * \operatorname{rand}(k=1,2), \quad x_{i, j}^{*}(t)=p_{i d}(t)(i=1,2, \ldots, N)$ and $x_{j}^{*}(t)=p_{g d}(t)$. Given that they are all constants, then equations (1) and (2) can be represented as follows:

$$
v_{i, j}(t+1)=\omega v_{i, j}(t)+\phi_{i, j}^{(1)}(t)\left(x_{i, j}^{\#}(t)-x_{i, j}(t)\right)+\phi_{i, j}^{(2)}(t)\left(x_{j}^{*}(t)-x_{i, j}(t)\right)
$$




$$
x_{i, j}(t+1)=x_{i j}(t)+v_{i j}(t+
$$

By eliminating the velocity parameters in formulas (15) and (16), we can get,

Let

$$
\begin{aligned}
& x_{i, j}(t+1)=\left(1+\omega-\phi_{i, j}^{(1)}-\phi_{i, j}^{(2)}\right) x_{i, j}(t)-\omega x_{i, j}(t-1)+\phi_{i, j}^{(1)} x_{i, j}^{\#}+\phi_{i, j}^{(2)} x_{j}^{*} \\
& A=\left[\begin{array}{ccc}
1+\omega-\varphi_{i, j}^{(1)}-\varphi_{i, j}^{(2)} & -\omega & \varphi_{i, j}^{(1)} x_{i, j}^{\#}+\varphi_{i, j}^{(2)} x_{j}^{*} \\
1 & 0 & 0 \\
0 & 0 & 1
\end{array}\right]
\end{aligned}
$$

Then the homogeneous matrix of equation (17) can be written:

$$
\left[\begin{array}{c}
x_{i, j}(t+1) \\
x_{i, j}(t) \\
1
\end{array}\right]=A \bullet\left[\begin{array}{c}
x_{i, j}(t) \\
x_{i, j}(t-1) \\
1
\end{array}\right]
$$

Thus, the eigenpolynomial of the coefficient matrix in formula (18) can be obtained by the following equation:

$$
(1-\lambda)\left(\omega-\lambda\left(1+\omega-\phi_{i, j}^{(1)}-\phi_{i, j}^{(2)}\right)+\lambda^{2}\right)
$$

It has three roots, viz. $\lambda=1, \alpha_{i, j}=\left(1+\omega-\phi_{i, j}^{(1)}-\phi_{i, j}^{(2)}+\gamma_{i, j}\right) / 2$ and $\beta_{i, j}=\left(1+\omega-\phi_{i, j}^{(1)}-\phi_{i, j}^{(2)}-\gamma_{i, j}\right) / 2$ respectively. Here $\gamma_{i, j}=\sqrt{\left(1+\omega-\phi_{i, j}^{(1)}-\phi_{i, j}^{(2)}\right)^{2}-4 \omega}$. So the convergence theorem of PSO is derived as follows. Viz., PSO is convergent if and only if $\max (\|\alpha\|,\|\beta\|) \leq 1$. Further, the corollary of the particle's velocity convergence is obtained, i.e., if PSO is convergent, then the particle's velocity decreases to zero continuously or holds just as its initial value during the whole searching process.

\subsection{Convergence Analysis with Difference Equation}

The convergence analysis of PSO is discussed in [13] by using difference equation. To begin with, simplifying the problem to be a one-dimensional space, assuming the best previous position of each particle and the best current position of the swarm are fixed, and denoted by $p_{b}$ and $g_{b}$, respectively. $c_{0}, c_{1}$ and $c_{2}$ are constants. Note that $c_{0}$, actually, is equivalent to the inertia weight $\omega$ in equation (1). Then the equations (1) and (2) can be simplified as follows:

$$
\begin{aligned}
& v(k+1)=c_{0} v(k)+c_{1}\left(p_{b}-x(k)\right)+c_{2}\left(g_{b}-x(k)\right) \\
& x(k+1)=x(k)+v(k+
\end{aligned}
$$

As a result, $x(k+2)=\left(c_{0}-c_{1}-c_{2}+1\right) x(k+1)-c_{0} x(k)+c_{1} p_{b}+c_{2} g_{b}$, that is to say,

$$
x(k+2)+\left(-c_{0}+c_{1}+c_{2}-1\right) x(k+1)+c_{0} x(k)=c_{1} p_{b}+c_{2} g_{b}
$$

Obviously, equation (22) is a non-homogeneous difference equation with a second order constant coefficient. Its characteristic equation is $\lambda^{2}+\left(-c_{0}+c_{1}+c_{2}-1\right) \lambda+c_{0}=0$. There exist three cases for solving it. 
(1) If $\Delta=\left(-c_{0}+c_{1}+c_{2}-1\right)^{2}-4 c_{0}=0$, then $\lambda=\lambda_{1}=\lambda_{2}=-\left(-c_{0}+c_{1}+c_{2}-1\right) / 2$,

$$
x(k)=\left[x(0)+\left(\frac{(1-c) x(0)+c_{0} v(0)+c_{1} p_{b}+c_{2} g_{b}}{\lambda}-x(0)\right) k\right] \lambda^{k} .
$$

(2) If $\Delta=\left(-c_{0}+c_{1}+c_{2}-1\right)^{2}-4 c_{0}>0$, then $\lambda_{1,2}=\left(c_{0}-c_{1}-c_{2}+1 \pm \sqrt{\Delta}\right) / 2$,

$$
\begin{aligned}
x(k) & =\frac{c_{1} p_{b}+c_{2} g_{b}}{c}+\frac{\lambda_{2} b_{1}-b_{2}}{\lambda_{2}-\lambda_{1}} \lambda_{1}^{k}+\frac{b_{2}-\lambda_{1} b_{1}}{\lambda_{2}-\lambda_{1}} \lambda_{2}^{k} . \\
\left(\text { Let } b_{1}\right. & \left.=x(0)-\left(c_{1} p_{b}+c_{2} g_{b}\right) / c, b_{2}=(1-c) x(0)+c_{0} v(0)+c_{1} p_{b}+c_{2} g_{b}-\left(c_{1} p_{b}+c_{2} g_{b}\right) / c\right)
\end{aligned}
$$

(3) If $\Delta=\left(-c_{0}+c_{1}+c_{2}-1\right)^{2}-4 c_{0}<0$, then $\lambda_{1,2}=\left(c_{0}-c_{1}-c_{2}+1 \pm i \sqrt{-\Delta}\right) / 2$,

$$
x(k)=\frac{c_{1} p_{b}+c_{2} g_{b}}{c}+\frac{\lambda_{2} b_{1}-b_{2}}{\lambda_{2}-\lambda_{1}} \lambda_{1}^{k}+\frac{b_{2}-\lambda_{1} b_{1}}{\lambda_{2}-\lambda_{1}} \lambda_{2}^{k} .
$$

$$
\left(\text { Let } b_{1}=x(0)-\left(c_{1} p_{b}+c_{2} g_{b}\right) / c, b_{2}=(1-c) x(0)+c_{0} v(0)+c_{1} p_{b}+c_{2} g_{b}-\left(c_{1} p_{b}+c_{2} g_{b}\right) / c\right)
$$

It is clear that, when $\mathrm{k}$ tends to infinity, the convergence conditions of the three cases described above are $\left\|\lambda_{1}\right\|<1$ and $\left\|\lambda_{2}\right\|<1$. And the convergent domain of the swarm is the delta-shaped region consists of $c_{0}<1, c_{1}+c_{2}>0,2 c_{0^{-}} c_{1^{-}} c_{2}+2>0$. Figure 2 graphs the convergent domain, which is the left-hand blank delta-shaped region.

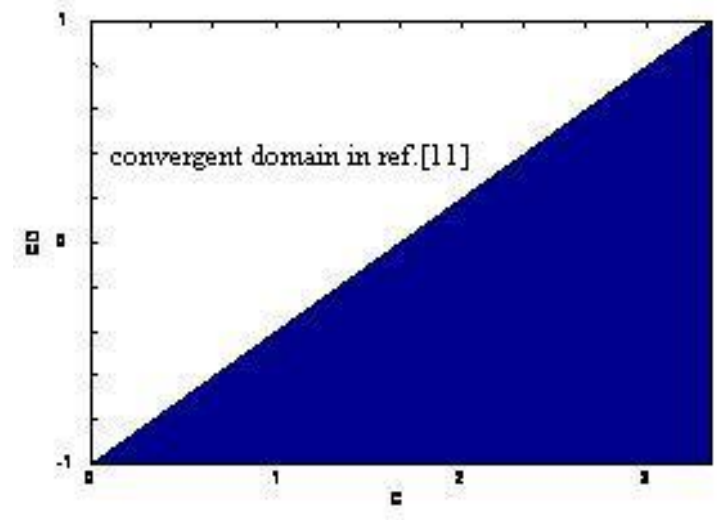

Figure 2. Convergent domain in ref. [11]

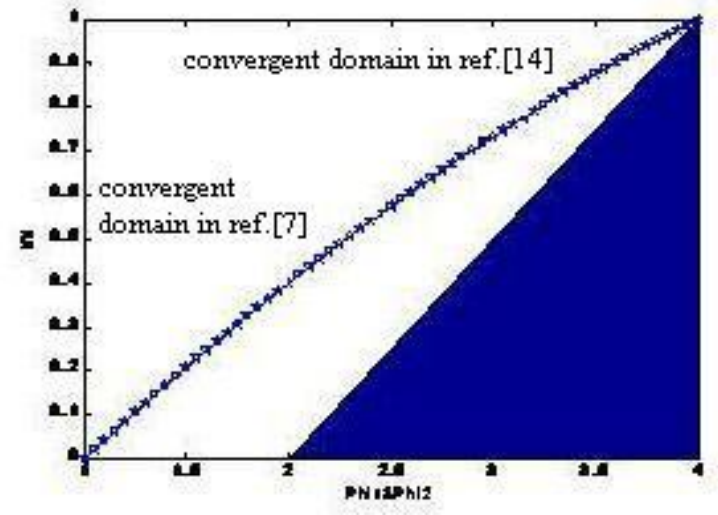

Figure 3. Convergent domains in refs. $[7,14]$

\subsection{Convergence Analysis with $Z$ Transformation}

The stability of the particle's trajectory is studied in [14] via $Z$ transformation. In order to analyze and express conveniently, first, the problem space is simplified to be one-dimension, and the $c_{1} \times r_{1}$ and $c_{2} \times r_{2}$ in equation (1) are denoted by $\Phi_{1}$ and $\Phi_{2}$, respectively. What is more, only one particle is considered here. And assuming the best previous position of each particle and the best current position of the swarm, denoted by $p_{i}$ and $p_{g}$ respectively are all fixed. Then the motion state of the $i$-th particle can be represented as follows:

$$
v_{i}(t+1)=\omega v_{i}(t)+\phi_{1}\left[p_{i}-x_{i}(t)\right]+\phi_{2}\left[p_{g}-x_{i}(t)\right]
$$




$$
x_{i}(t+1)=x_{i}(t)+v_{i}(t+1)
$$

Thus, the following equation can be derived via substituting between the equations (23) and (24).

$$
v_{i}(t+2)+\left(\phi_{1}+\phi_{2}-\omega-1\right) v_{i}(t+1)+\omega v_{i}(t)=0
$$

As can be seen from the formula (25), the process of the particle's velocity change is a second-order difference equation. Similarly, the process of the particle's position change can be obtained as follows, and it is also a second-order difference equation.

$$
x_{i}(t+2)=\left(1+\omega-\phi_{1}-\phi_{2}\right) x_{i}(t+1)-\omega x_{i}(t)+\phi_{1} p_{i}+\phi_{2} p_{g}
$$

(1) Executing $Z$ transformation on equation (25), then we can get,

$$
V_{i}(z)=\left(z^{2} v_{i}(0)+z v_{i}(1)+z\left(\phi_{1}+\phi_{2}-\omega-1\right) v_{i}(0)\right) /\left(z^{2}+z\left(\phi_{1}+\phi_{2}-\omega-1\right)+\omega\right)
$$

where $\Phi_{1}$ and $\Phi_{2}$ are stochastic numbers and they are supposed to be constants so as to simplify the analysis. Thus equation (27) will become a linear system and its characteristic equation is:

$$
z^{2}+z\left(\phi_{1}+\phi_{2}-\omega-1\right)+\omega=0
$$

Furthermore, substituting $z=(\mu+1) /(\mu-1)$ into equation (28) and executing bilinear transformation can lead to the following result,

$$
\left(\phi_{1}+\phi_{2}\right) \stackrel{2}{\mu}+(2-2 \omega) u+\left(2 \omega+z_{1} \phi-{ }_{2} \phi\right.
$$

From Routh criterion, the necessary and sufficient condition of a stable second-order linear system is that each coefficient of the characteristic equation is a positive constant. So the stable condition of formula (25) can be derived as below:

$$
\left\{\begin{array}{c}
\varphi_{1}+\varphi_{2}>0 \\
1-\omega \geq 0 \\
2 \omega+2-\varphi_{1}-\varphi_{2} \geq 0
\end{array}\right.
$$

Due to $\Phi_{1}$ and $\Phi_{2}$ are positive real numbers, and the $p_{i}$ and $p_{g}$ are assumed to be constants previously. Hence, the stable condition described by equation (30) can be further simplified as follows:

$$
\left\{\begin{array}{c}
1-\omega \geq 0 \\
2 \omega+2 \geq \varphi_{1}+\varphi_{2}
\end{array}\right.
$$

When the strict unequal condition in (31) is satisfied, the velocity of a single particle will tend to zero.

(2) Executing $\mathrm{Z}$ transformation on equation (26), as a result,

$$
X_{i}(z)=\left(z^{3} x_{i}(0)+z^{2}\left(x_{i}(1)+(\varsigma-1) x_{i}(0)\right)+z\left(\phi_{1} p_{i}+\phi_{2} p_{g}-\varsigma x_{i}(0)-x_{i}(1)\right)\right) /\left(\left(z^{2}+\varsigma z+\omega\right)(z-1)\right)
$$

where $\zeta=\varphi_{1}+\varphi_{2}-1-\omega$. Then the characteristic equation of the linear system corresponding to equation (32) is given below, 


$$
\left(z^{2}+z\left(\phi_{1}+\phi_{2}-\omega-1\right)+\omega\right)(z-1)=0
$$

Similarly, substituting $z=(\mu+1) /(\mu-1)$ into equation (33) and executing bilinear transformation can lead to the result,

$$
\left(\phi_{1}+\phi_{2}\right) \mu^{2}+(2-2 \omega) \mu+\left(2 \omega+2-\phi_{1}-\phi_{2}\right)=0
$$

From equations (34) and (29), it is easy to see that there are same zero points for the characteristic equation corresponding to equations (32) and (27). Therefore, the stable conditions are same. i.e., the stable condition of the particle's position change also is equation (31). When this condition is met, the position of a single particle will tend to be $\left(\varphi_{1} p_{i}+\varphi_{2} p_{g}\right)$ $/\left(\varphi_{1}+\varphi_{2}\right)$.

Figure 3 graphs the comparison result of convergent domain in [14] and [7]. Note that the left-hand blank trapezoidal domain is derived form equation (33) in [14], which represents the convergent domain of a single particle, whereas the solid line is derived from the equation $K=2 /\left|2-\tau-\sqrt{\tau^{2}-4 \tau}\right|$ in [7]. Obviously, much larger convergent domain can be obtained by [14] than that by [7].

\subsection{Other Convergence Analyses}

Recently, Liu et al., [15] discuss the swarm intelligent model, i.e., the particle swarm based on its iterated function system. The dynamic trajectory of the particle is described based on single individual and the swarm algorithm is proved to be converged with a probability of one towards the global optimal. Fang et al., [16] propose quantum-behaved particle swarm optimization (QPSO) algorithm and discuss the convergence of QPSO within the framework of random algorithm's global convergence theorem. Meanwhile, an improved particle swarm optimization algorithm is presented by Liang et al., [17] and its convergence is analyzed in a simplified one dimension. In addition, the convergence time of particle swarm optimization is analyzed on the facet of particle interaction [18], in which the theoretical analysis is conducted on the social-only model of PSO instead of on common models in practice. The theoretical results reveal the relationship between the convergence time and the level of convergence as well as the relationship between the convergence time and the swarm size. In the most recent years, Ren et al., [19] analyze the global convergence of PSO algorithm, in which the one-step transition probabilities of particle velocity and particle position are calculated as well as several properties about this Markov chain are investigated. And it is proved that the particle state space is non-recurrent and the PSO algorithm is not global convergent from the viewpoint of the transition probability.

\section{Conclusion and Expectation}

From the convergence analyses of PSO reviewed above, we can easily draw the following important conclusions.

- In Subsection 3.1, the use of the constriction coefficient $K$ can be viewed as a recommendation to the particle to "take smaller steps", which makes the particles move toward the point $P\left(v=0, x=\left(c_{1} p_{i d}+c_{2} p_{g d}\right) /\left(c_{1}+c_{2}\right)\right)$ and tend to convergence at last. The convergence mentioned here obviously implies that the particle's velocity $=0$. But the position $x$ obtained here is not necessarily the convergent point that is wanted, particularly if the system is too constricted. 
- In Subsection 3.2, the relation between the convergence and the variance of the population's fitness is strictly derived by using limit. To a certain extent, it reflects the dynamic performance of PSO. However, it is difficult to distinguish premature convergence from global convergence only by the variance of the population's fitness is zero.

- In Subsection 3.3, the relation between the inertia weight and the particle's velocity as well as its influence on the convergence of PSO is illustrated. As can be seen from the previous description, the problem space is assumed to be one-dimensional and only one particle is considered when the convergence of PSO is analyzed. In actual fact, there are a large number of particles and the real-world problems usually lie in three-dimensional space.

- In Subsection 3.4, $\phi_{i, j}^{(k)}(k=1,2)$ corresponds to the $c_{i *}$ rand $(i=1,2)$ in equation (1). Whether the acceleration coefficients $c_{i}(i=1,2)$ are fixed or linearly change as the iterative number increases [20], $c_{i *}$ rand $(i=1,2)$ are always variables. Meanwhile, $x_{i, j}^{\#}$ and $x_{j}^{*}$ correspond to $p_{i d}(t)$ and $p_{g d}(t)$ respectively. They are all dynamic variables during the evolution process. However, all of them are supposed to be constants in the literature.

- In Subsection 3.5, when the convergence of PSO is analyzed by using difference equation, like other methods used in above literatures, all of the variables appearing in equations (1) and (2) are assumed to be constants, and the problem space is viewed as one-dimensional.

- In Subsection 3.6, when the stability of the particle's trajectory is analyzed via $Z$ transformation, the problem is simplified to be a second-order linear system under certain assumptions, and its stable condition is derived from the Routh criterion.

In the light of the analyses given above, it is clear that the convergence analyses of PSO studied by many researchers are all under certain rigid assumptions. Although these assumptions can simplify the problem models and then the models can be easily studied and analyzed by researchers. They, to a certain degree, restrict the convergence study of the PSO systems, even the establishment of the PSO's theoretical foundations.

PSO has been accepted widely as a potential global optimizing algorithm because of its convenience of realization and low constraints on the environment and objective functions, but there is still a great space for the research of the algorithm itself. So far, the mathematical proofs of PSO's convergence, convergent velocity, parameter selection and robustness have not been proposed perfectly. Hence, how to study and analyze PSO by the ideas of limit, probability, evolution and topology in order to reflect the mechanism of how PSO works, which is also a highly desired subject that should be paid much attention to by PSO researchers.

\section{Acknowledgements}

The work is supported by the National Natural Science Foundation of China (No.61035003, No.61072085, No.60933004, No.60903141), the National Program on Key Basic Research Project (973 Program) (No.2013CB329502), the National High-tech R\&D Program of China (863 Program) (No.2012AA011003), the National Science and Technology Support Program of China (2012BA107B02), the Natural Science Foundation Research of the Department of Science and Technology of Shaanxi Province (No.SJ08-ZT13,No.2011K09-35) 
and the Science and Research Planning Project of the Education Department of Shaanxi Province (No.09JK335).

\section{References}

[1] J. Kennedy and R. Eberhart, "Particle swarm optimization", Proceedings of the IEEE International Conference on Neural Networks, vol. 4, (1995), pp. 1942-1948.

[2] Y. Shi and R. Eberhart, "A modified particle swarm optimizer", Proceedings of the IEEE International Conference on Evolutionary Computation, (1998), pp. 69-73.

[3] J. Kennedy and R. Mendes, "Population structure and particle swarm performance", Proceedings of the IEEE Congress on Evolutionary Computation (CEC'02), vol. 2, (2002), pp. 1671-1676.

[4] P. Angeline, "Evolutionary optimization versus particle swarm optimization: Philosophy and Performance differences", Proceedings of the 7th International Conference on Evolutionary Programming VII (EP'98), (1998), pp. 601-610.

[5] X. Yao, "Evolving artificial neural networks", Proceedings of the IEEE International Conference on Neural Networks and Brain, vol. 87, no. 9, (1999), pp. 1423-1447.

[6] T. Sousa, A. Silva and A. Neves, "Particle swarm based data mining algorithms for classification tasks", Parallel Computing-Special issue: Parallel and nature-inspired computational paradigms and applications, vol. 30, no. 5, (2004), pp. 767-783.

[7] C. Maurice and J. Kennedy, "The particle swarm-explosion, stability and convergence in a multidimensional complex space", IEEE Transactions on Evolutionary Computation, vol. 6, no. 1, (2002), pp. 58-73.

[8] Z. Lu, Z. Hou and J. Du, "Particle swarm optimization with adaptive mutation", Acta Electronica Sinica, vol. 32, no. 3, (2004), pp. 416-420.

[9] F. van den Bergh, "An Analysis of particle swarm optimizers", Department of Computer Science, University of Pretoria, South Africa, (2002), pp.81-83.

[10] L. Zhang, H. Yu, D. Chen and S. Hu, "Analysis and improvement of particle swarm optimization algorithm", Information and Control, vol. 33, no. 5, (2004), pp. 513-517.

[11] F. van den Bergh, "An analysis of particle swarm optimizers", Pretoria: University of Pretoria, (2006), pp.78-85.

[12] H. Liu, X. Wang and G. Tan, "Convergence analysis of particle swarm optimization and its improved algorithm based on chaos", Control and Decision, vol. 21, no. 6, (2006), pp. 636-640.

[13] S. Gao, K. Tang, X. Jiang and J. Yang, "Convergence analysis of particle swarm optimization algorithm", Science Technology and Engineering, vol. 6, no. 12, (2006), pp. 1625-1627.

[14] N. Li, D. Sun, T. Zou, Y. Qin and Y. Wei, “An analysis for a particle's trajectory of PSO based on difference equation”, Chinese Journal of Computers, vol. 29, no. 11, (2006), pp. 2052-2061.

[15] H. Liu, A. Abraham and V. Snasel, "Convergence analysis of swarm algorithm", In: Proceedings of the International Conference on Nature \& Biologically Inspired Computing (NaBIC'09), (2009), pp. 1714-1719.

[16] W. Fang, J. Sun, Z. Xie and W. Xu, "Convergence analysis of quantum-behaved particle swarm optimization algorithm and study on its control parameter", Acta Physica Sinica, vol. 59, no. 6, (2010), pp. 3686-3694.

[17] S. Liang, S. Song, L. Kong and J. Cheng, "An improved particle swarm optimization algorithm and its convergence analysis", In: Proceedings of the 2nd International Conference on Computer Modeling and Simulation (ICCMS'10), (2010), pp. 138-141.

[18] C. Chen and Y. Chen, "Convergence time analysis of particle swarm optimization based on particle interaction”, Advances in Artificial Intelligence, (2011), pp. 1-7.

[19] Z. Ren, J. Wang and Y. Gao, "The global convergence analysis of particle swarm optimization algorithm based on Markov chain", Control Theory \& Applications, vol. 28, no. 4, (2011), pp. 462-466.

[20] A. Ratnaweera, S. Halgamuge and H. Watson, "Self-organizing hierarchical particle swarm optimizer with time-varying acceleration coefficients", IEEE Transactions on Evolutionary Computation, vol. 8, no. 3, (2004), pp. 240-255. 
International Journal of Grid and Distributed Computing Vol.6, No.6 (2013) 